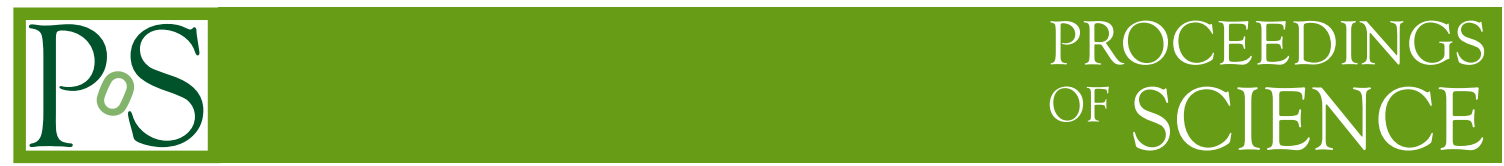

\title{
Frontier Research in Astrophysics 2014 Concluding Remarks
}

\author{
René Hudec ${ }^{1,2 * \dagger}$ \\ ${ }^{1}$ Czech Technical University in Prague, Faculty of Electrical Engineering, \\ Technicka 2, CZ 16000 Prague, Czech Republic \\ ${ }^{2}$ Astronomical Institute of the Academy of Sciences of the Czech Republic \\ Fricova 298 - CZ 25165 Ondrejov, Czech Republic \\ E-mail: rene.hudecegmail.com
}

This paper contains the printed version of the concluding remarks I gave at the Frascati Workshop on Frontier Research in Astrophysics, held in Mondello, Italy, from May 26-31, 2014.

Frontier Research in Astrophysics,

26-31 May 2014

Mondello (Palermo), Italy

* Speaker.

${ }^{\dagger}$ A footnote may follow. 


\section{Introduction}

The idea of this international workshop is to bring together researchers (astrophysicists, particle physicists, and astronomers) who are involved in various topics at the forefront of modern astrophysics and particle physics. The workshop discussed the most recent experimental and theoretical results in order to advance our understanding of the physics governing our Universe.

I believe the goals of the workshop were achieved by a synergy between data from groundbased and space-based experiments, as well as results from theoretical developments. This work at the forefront of science is very likely to result in scientific papers of significant import.

Hence, the main purpose of the workshop, which was to discuss in a unique and collaborative setting a broad range of topics in modern astrophysics, from the Big Bang to Planets and Exoplanets, has been accomplished. Without doubt, the scope of the workshop has provided a suitable framework for each participant who (while obviously not involved in all the topics discussed), was be able to acquire a general view of the main experimental and theoretical results currently obtained.

Such an up-to-date view of the current research on cosmic sources, as presented by workshop speakers and participants, can help guide future research projects by the participants, and surely will encourage collaborative efforts across various topical areas of research.

\section{A very few subjective comments.}

In my opinion, the 2014 Frascati Workshop in Mondello represents another confirmation of Franco Giovannelli's "Vulcano Theorem" (more details are given in Professor Franco Giovannelli's concluding talk). Apart from this theorem, the keys for a productive workshop are very obvious: a nice environment, especially delicious dining, and staying together. This allows contacts and discussions among participants not only during the scientific sessions, but for the whole duration of the conference.

In comparing the previous Frascati Vulcano/Mondello workshops with the 2014 workshop, it appears to me that this was innovative in the sense that it covered a wider range of scientific fields than before, even while focusings on the frontiers of astrophysics. Including a wider range of topics is definitely a positive move. For example in x-ray astronomy and astrophysics, there are communities working in solar, and even planetary and cometary research, and bringing them together with the galactic and extragalactic $\mathrm{x}$-ray astronomy community is surely beneficial for all.

Additionally, there has been a strong participation both of leaders as well as young scientists in these fields. Most importantly, there have been many occasions for young researchers to meet famous scientists they had known previously only from the literature, both in the question periods and in informal settings.

In addition, I note the very high quality of the invited and review talks - this is not trivial as we face the problem of the declining quality of such talks at numerous other conferences.

In my opinion, the following selected pictures taken during the workshop will better illustrate the scope, atmosphere, and success of the conference better than words. 

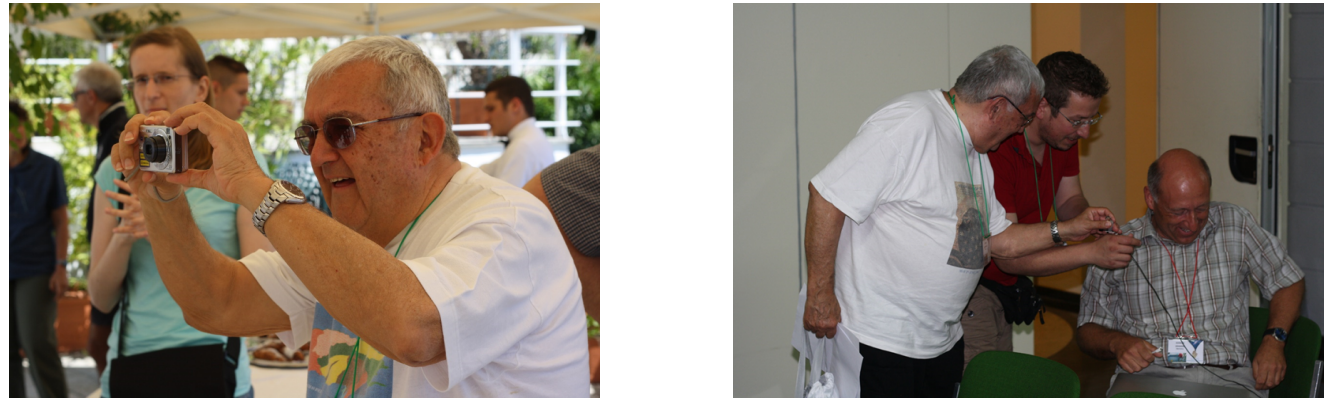

Figure 1: Conference organizers
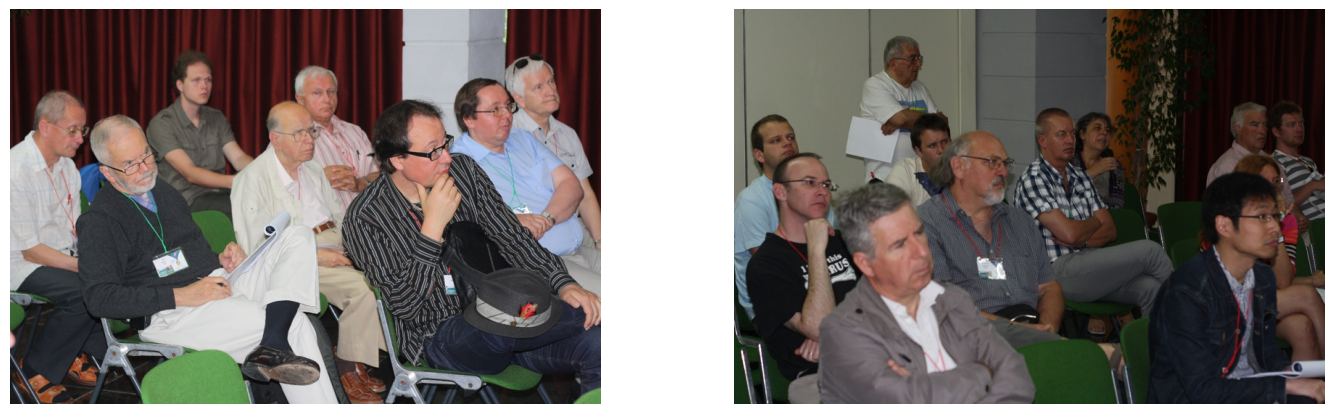

Figure 2: Sessions
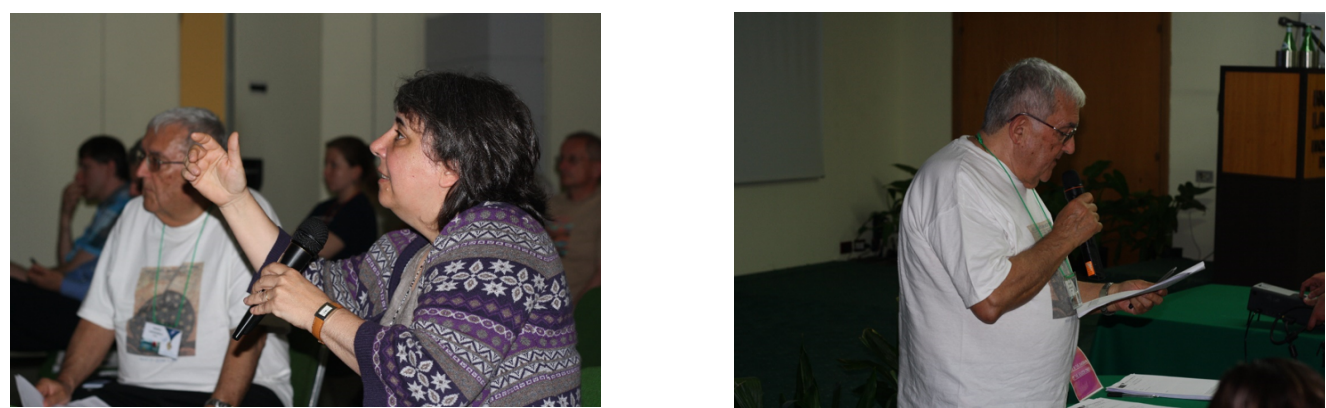

Figure 3: Sessions II

\section{Conclusions}

In conclusion, I am confident that the goal of the workshop was achieved by the synergy between data from ground-based and space-based experiments as well as results from theoretical work. This work on the forefront of the scientific enterprise is likely to result in high-impact scientific research. Hence, the main purpose of the workshop (namely, to discuss in a unique and collaborative setting a broad range of topics in modern astrophysics) had been achieved. I also believe it will encourage collaborative efforts across various topical areas of research.

\section{Acknowledgments}

This paper and author participation in the conference was supported by the grant GA CR 13$33324 \mathrm{~S}$. 

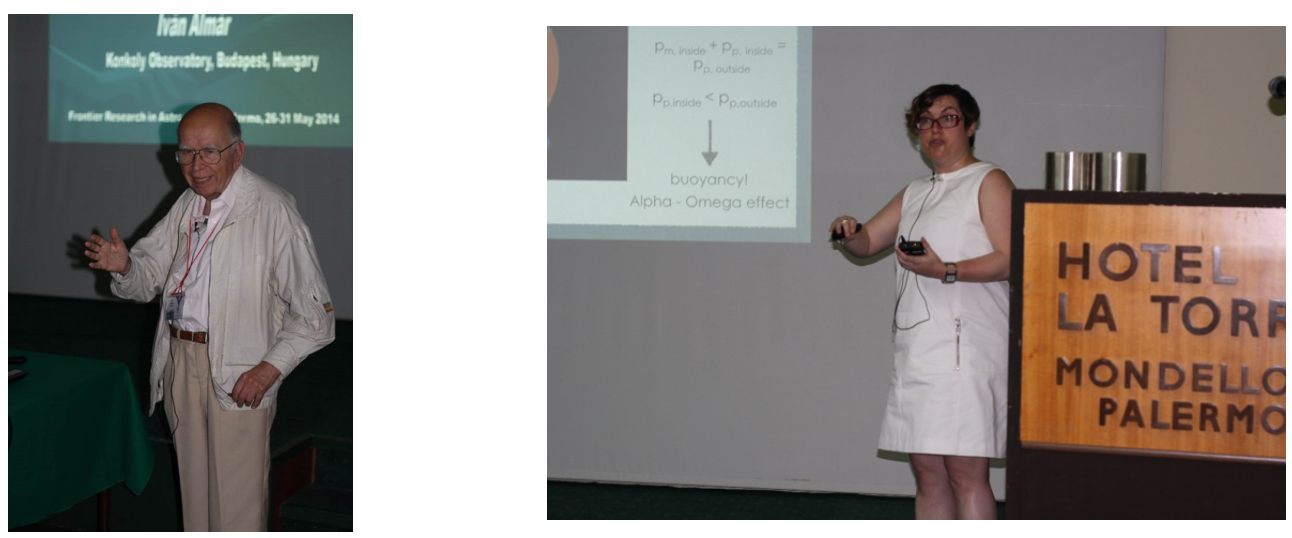

Figure 4: Talks
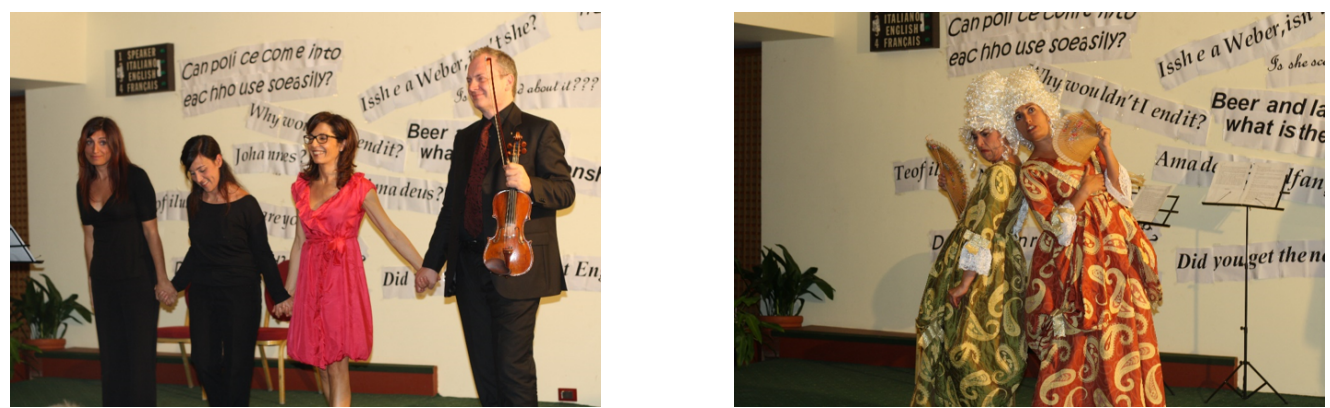

Figure 5: Concert
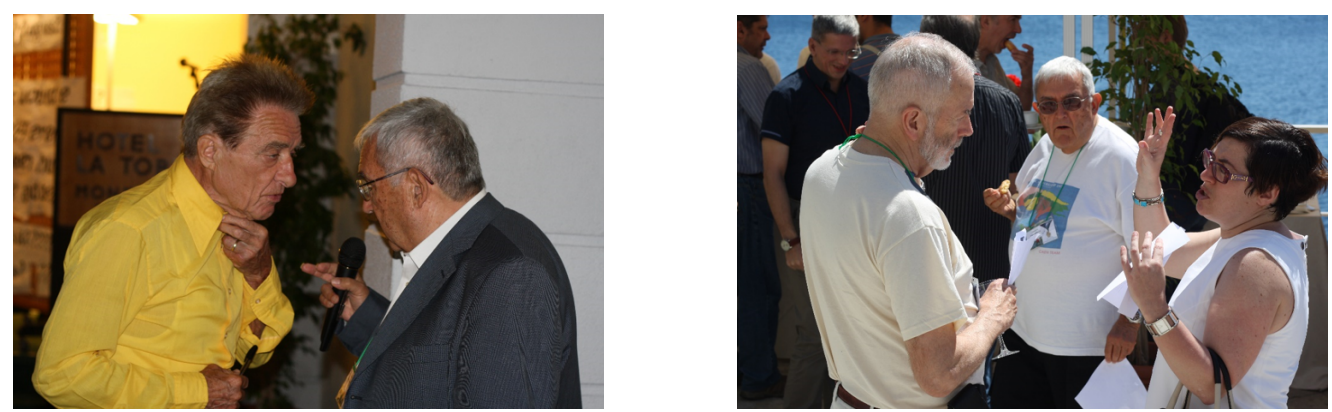

Figure 6: Discussions
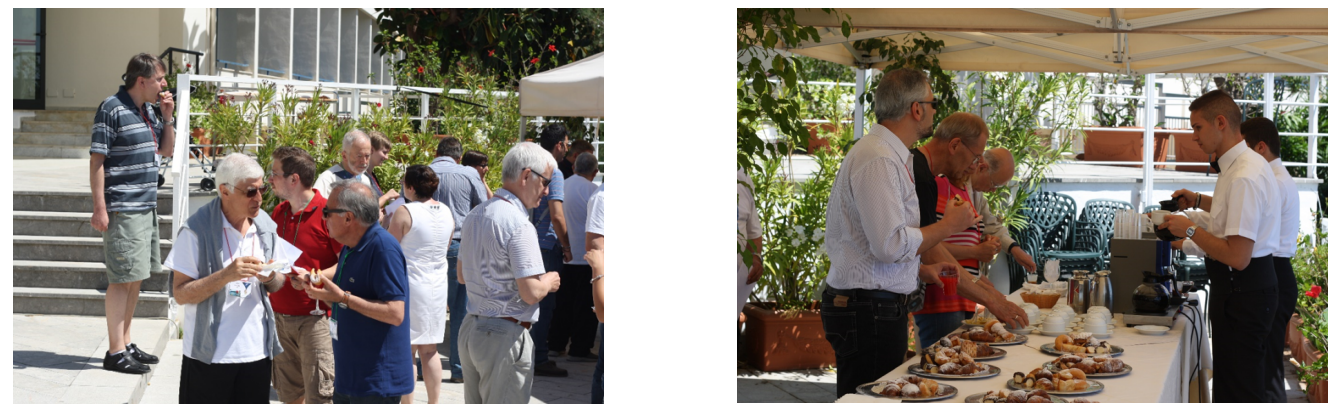

Figure 7: Coffee breaks 

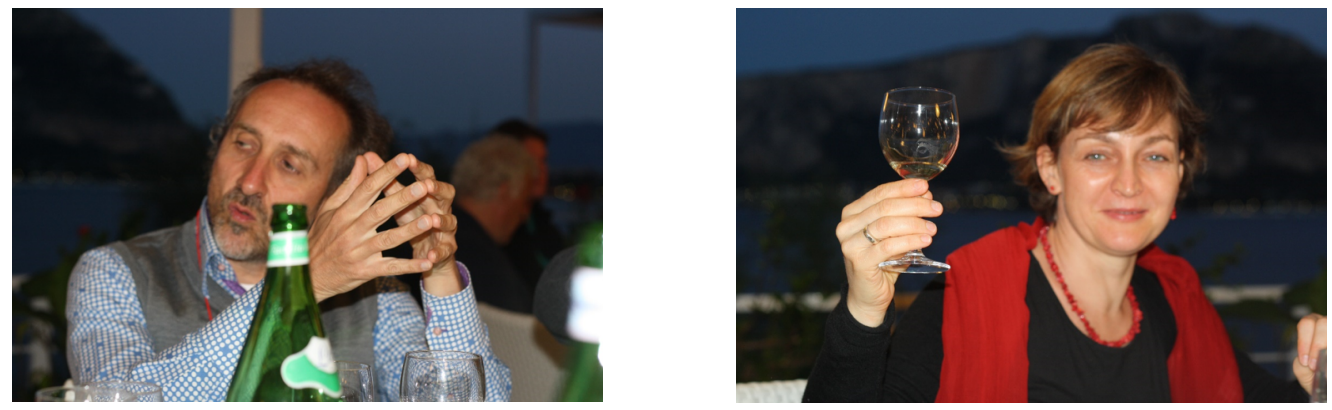

Figure 8: Dining
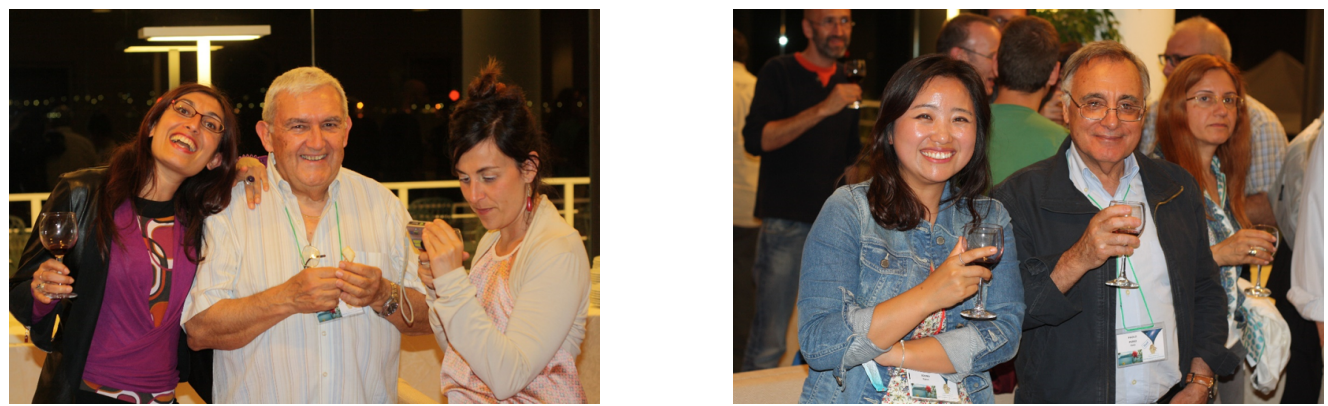

Figure 9: Etruscan wine party
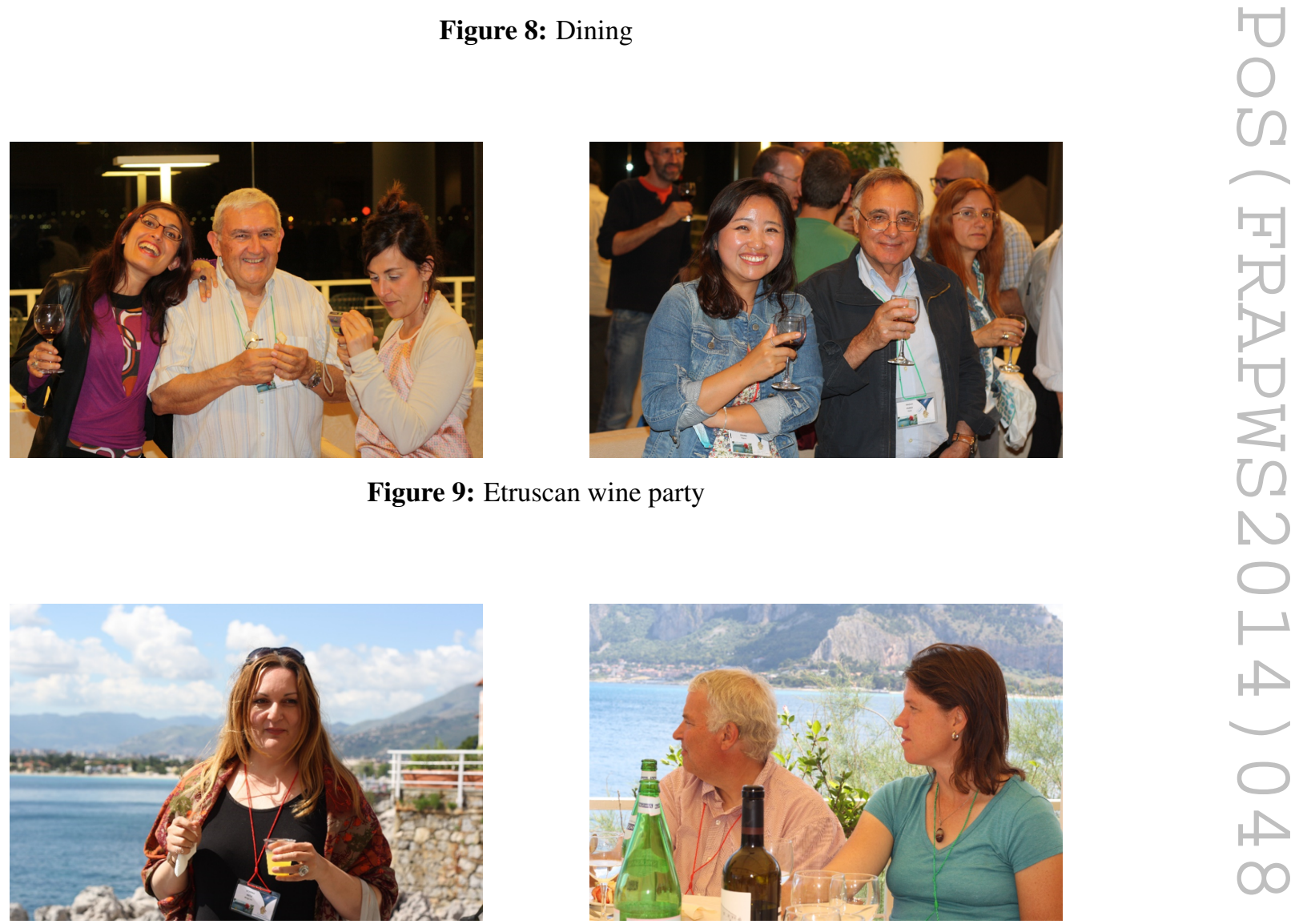

Figure 10: Enjoying Mondello
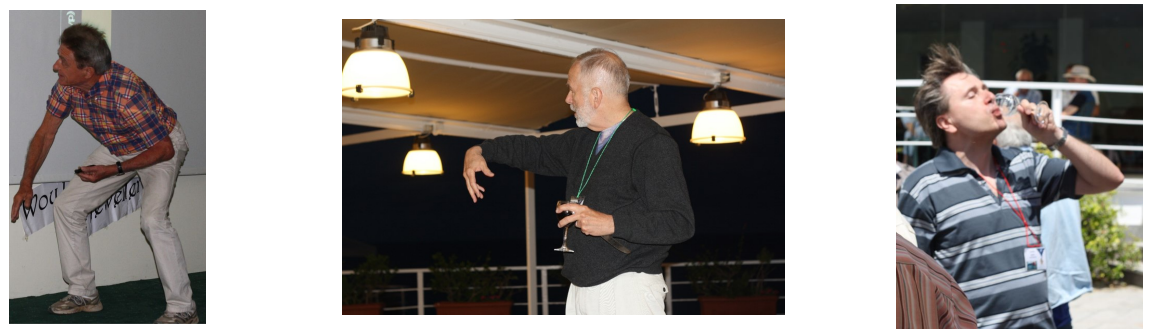

Figure 11: Conference highlights 